(c) American Dairy Science Association, 2003.

\title{
A Simple Method for Weighted Bending of Genetic (Co)variance Matrices
}

\author{
H. Jorjani, ${ }^{*}$ L. Klei, $†$ and U. Emanuelson* \\ *Interbull Centre, Department of Animal Breeding and Genetics, Swedish University of Agricultural Sciences, 75007 Uppsala, Sweden \\ †Holstein Association USA, Inc., 1 Holstein Place, Brattleboro, VT 05301-0808
}

\begin{abstract}
Due to computational demand, elements of genetic correlation matrices may have been estimated separately and then combined together into a single correlation matrix at a later stage. Because these matrices should be positive definite (PD) a statistical method commonly known as "bending" is used to make them positive definite. The conventional bending method ignores the reliability of different correlations and may subject any of them to change in order to make a positive definite correlation matrix. A simple method to obtain a weighted bended matrix to be used in animal breeding applications is proposed, and the implementation of the method is demonstrated by an example.
\end{abstract}

(Key words: genetic correlation matrix, covariance matrices, weighted bending)

Abbreviation key: PD = positive definite.

\section{INTRODUCTION}

In many animal breeding applications, matrices of genetic correlations for a large number of traits are needed. Estimation of breeding values for bulls in the context of international genetic evaluation of dairy cattle populations and construction of total merit indices are just two examples. These matrices should fulfill certain mathematical and statistical criteria, one of which is that they should be positive definite (PD). Matrices need to be PD because their Cholesky decompositions or inverses are needed. However, because estimation of genetic correlations is sometimes very computationally demanding, genetic correlations are usually estimated for only a subset of all traits of interest. In many occasions only bivariate estimates of correlations are available. Further, in some occasions genetic correlations are not estimated at all and are simply taken from literature. Irrespective of the way the individual genetic correlation values are obtained, these estimated

Received August 12, 2002.

Accepted September 16, 2002.

Corresponding author: Hossein Jorjani; e-mail: Hossein.Jorjani@ hgen.slu.se. correlations are combined to create a large matrix for all traits. The resulting matrix of all traits is almost always non-PD and should be converted to a PD matrix by a process commonly known as bending.

The term "bending" has been used in animal breeding literature to describe two different, although related, procedures. In the original meaning (e.g., Hayes and Hill, 1981; Meyer and Hill, 1983; Árnason, 1984; Meuwissen and Kanis, 1988; and Essl, 1991), the term "bending" was used in the context of selection index theory and for optimization of the selection response, and positive definiteness of correlation matrices has not been an important issue. The other meaning of the term "bending" directly addresses the issue of positive definiteness of matrices, and although it has been used colloquially among animal breeders, it is only recently that it has started to appear in the literature (e.g., Jorjani et al., 2002; and Henshall and Meyer, 2002).

From a matrix algebra point of view the bending process, defined in this paper as "unweighted" bending, is quite simple. However, it is not desirable to use an unweighted bending method because it is indiscriminate to all elements of the correlation matrix and both highly reliable elements and less reliable ones are equally likely to be changed in the process. Here we present a simple method to obtain "weighted" bended matrices that minimizes changes to the more reliable correlations.

\section{METHOD}

Bending is an iterative process of updating the covariance or correlation matrix as follows. Let $\mathbf{V}$ be a symmetric non-PD matrix of (co)variances. Further, let $\mathbf{W}$ be a matrix of weighting factors for elements of $\mathbf{V}$. The bending process then would be as follows:

1. Determine matrix of eigenvectors, $\mathbf{U}_{n}$, and diagonal matrix of eigenvalues, $\mathbf{D}_{\mathrm{n}}$, of $\mathbf{V}$. Hence, $\mathbf{V}_{\mathrm{n}}=$ $\mathbf{U}_{\mathrm{n}} \mathbf{D}_{\mathrm{n}} \mathbf{U}_{\mathrm{n}}{ }^{\prime}$, where $\mathrm{n}$ denotes iteration number;

2. Replace $\mathbf{D}_{\mathrm{n}}$ with $\boldsymbol{\Delta}_{\mathrm{n}}$, where $\delta_{\mathrm{i}, \mathrm{i}}=\varepsilon$, for $\mathrm{d}_{\mathrm{i}, \mathrm{i}}<\varepsilon$, and $\delta_{\mathrm{i}, \mathrm{i}}=\mathrm{d}_{\mathrm{i}, \mathrm{i}}$, otherwise. Set the value of $\varepsilon$ to a small positive real number;

3. Calculate a new covariance matrix: $\mathbf{V}_{\mathrm{n}+1}=\mathbf{V}_{\mathrm{n}}-\left[\mathbf{V}_{\mathrm{n}}-\right.$ $\left.\mathbf{U}_{\mathrm{n}} \boldsymbol{\Delta}_{\mathrm{n}} \mathbf{U}_{\mathrm{n}}{ }^{\prime}\right] \odot \mathbf{W}$ (where $\odot$ is the Hadamard product);

4. Repeat until $\mathbf{V}_{n+1}$ is positive definite. 
If we let $\mathbf{W}=a \mathbf{C}$, where $a$ is any scalar and $\mathbf{C}$ is a matrix of weighting coefficients, then it can easily be seen that $a=1.0$ and $\mathbf{C}=\mathbf{J}$, i.e., $\mathbf{W}=\mathbf{J}$, leads to unweighted bending of the (co)variance matrix.

Extension to correlation matrices (R) puts some constraints on the algorithm described above because of some special properties of $\mathbf{R}$, namely that the trace of $\mathbf{R}(\operatorname{tr}(\mathbf{R}))$, and consequently $\operatorname{tr}(\mathbf{D})$, are equal to the order of $\mathbf{R}$ and the diagonal elements of $\mathbf{R}$ (and $\mathbf{U} \boldsymbol{\Delta} \mathbf{U}^{\prime}$ ) must be equal to unity. To accommodate these properties, replace steps 2 and 3 with the following steps:

2a) Replace $\mathbf{D}_{\mathrm{n}}$ with $\mathbf{D}^{*}{ }_{\mathrm{n}}$, where $\mathrm{d}^{*}{ }_{\mathrm{i}, \mathrm{i}}=2 \varepsilon$, for $\mathrm{d}_{\mathrm{i}, \mathrm{i}}<$ $\varepsilon$ and $\mathrm{d}_{\mathrm{i}, \mathrm{i}}=\mathrm{d}_{\mathrm{i}, \mathrm{i}}$, otherwise;

2.1a) Replace $\mathbf{D}^{*}{ }_{\mathrm{n}}$ with $\boldsymbol{\Delta}_{\mathrm{n}}$, where $\delta_{\mathrm{i}, \mathrm{i}}=\mathrm{d}^{*}{ }_{\mathrm{i}, \mathrm{i}}(\operatorname{tr}(\mathbf{D}) /$ $\operatorname{tr}\left(\mathbf{D}^{*}\right)$ );

3a) Calculate a new correlation matrix: $R^{*}{ }_{n+1}=\mathbf{R}_{n}$ $-\left[\mathbf{R}_{\mathrm{n}}-\mathbf{U}_{\mathrm{n}} \boldsymbol{\Delta}_{\mathrm{n}} \mathbf{U}_{\mathrm{n}}{ }^{\prime}\right] \odot \mathbf{W}$

3.1a) Replace $\mathbf{R}^{*}{ }_{n+1}$ with $\mathbf{R}_{n+1}$, where $r_{i, j}=r^{*}{ }_{i, j} /$ sqrt $\left(r_{i, i}^{*} r_{j, j}\right)$.

The method described above for unweighted bending of correlation matrices is very similar to the method described by Klei et al. (1988) except they added a certain value to all eigenvalues in order to make the smallest one equal to $\varepsilon$.

\section{EXAMPLE}

To demonstrate the effects of choosing different values for the $\mathbf{W}$ matrix the following matrices will be used (Jorjani et al., 2001). Let the starting covariance matrix, $\mathbf{V}_{0}$, be the symmetric matrix with values equal to the diagonal and upper diagonal elements of the matrix below. Elements of the corresponding correlations matrix, $\mathbf{R}_{0}$, are in the lower diagonal and will be used later (The same notation is used for the other matrices in this paper unless noted otherwise).

$$
\left[\begin{array}{ccccc}
100 & 95 & 80 & 40 & 40 \\
0.95 & 100 & 95 & 80 & 40 \\
0.80 & 0.95 & 100 & 95 & 80 \\
0.40 & 0.80 & 0.95 & 100 & 95 \\
0.40 & 0.40 & 0.80 & 0.95 & 100
\end{array}\right]
$$

and let the matrix of weighting factors, $\mathbf{W}$, be either equal to $\mathbf{J}$ or equal to the reciprocal of number of animals used in the estimation of covariances (as an approximation to the reliability of estimates) shown in the following matrix:

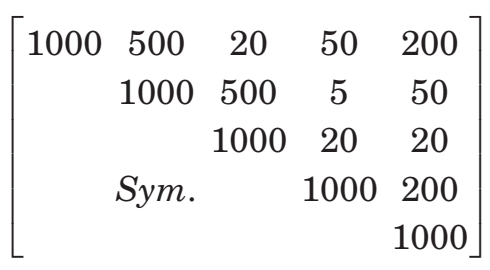

By using $\mathbf{W}=\mathbf{J}$ and $\varepsilon=10^{-4}$ a PD covariance matrix, $\mathbf{V}_{4}$ (unweighted), and its corresponding correlation matrix, $\mathbf{R}_{4}$ (unweighted), are obtained after four rounds of iteration (shown in Matrix [3]).

$$
\left[\begin{array}{ccccc}
103.2 & 90.8 & 79.5 & 44.5 & 37.1 \\
0.87 & 106.5 & 94.2 & 74.1 & 44.5 \\
0.77 & 0.90 & 102.3 & 94.2 & 79.5 \\
0.42 & 0.70 & 0.90 & 106.5 & 90.8 \\
0.36 & 0.42 & 0.77 & 0.87 & 103.2
\end{array}\right]
$$

However, using the matrix of weighting factors equal to the reciprocal of number of animals leads to a PD covariance matrix, $\mathbf{V}_{5018 \text { (weighted), and its corresponding }}$

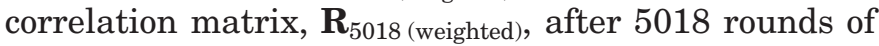
iteration, shown in the matrix below.

$$
\left[\begin{array}{ccccc}
100.2 & 94.5 & 82.9 & 43.6 & 39.2 \\
0.94 & 100.6 & 94.0 & 60.0 & 45.9 \\
0.83 & 0.93 & 100.7 & 84.9 & 73.1 \\
0.44 & 0.60 & 0.85 & 100.3 & 94.2 \\
0.39 & 0.46 & 0.73 & 0.94 & 100.2
\end{array}\right]
$$

Comparison of the weighted matrices $\left(\mathbf{V}_{5018 \text { (weighted) }}\right.$ and $\mathbf{R}_{5018 \text { (weighted) }}$ ) with their corresponding unweighted matrices ( $\mathbf{V}_{4}$ (unweighted) and $\mathbf{R}_{4}$ (unweighted), respectively) show that the weighted bending minimizes the changes to more reliable estimates at the expense of larger changes to less reliable ones. For example, the more reliable element $(1,2)$ has changed from 0.95 to 0.94 (instead of 0.87 ) and the less reliable element $(2,4)$ has changed from 0.80 to 0.60 (instead of 0.70 ), i.e., the proposed algorithm does exactly what we intuitively wanted it to do.

Starting with the correlation matrix $\mathbf{R}_{0}$ the algorithm for correlations (steps 2a to 3.1a) leads to an unweighted

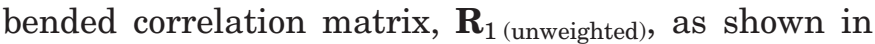
Matrix [3] after one round of iteration and to a weighted bended correlation matrix $\left(\mathbf{R}_{1715 \text { (weighted) }}\right)$ after 1715 rounds of iteration, as shown in the following matrix:

$$
\left[\begin{array}{llllll}
1.00 & 0.94 & 0.78 & 0.38 & 0.39 \\
& 1.00 & 0.93 & 0.49 & 0.41 \\
& & 1.00 & 0.74 & 0.62 \\
& & \text { Sym. } & & 1.00 & 0.93 \\
& & & & 1.00
\end{array}\right]
$$

Using correlation matrices in the bending process leads understandably to different results than what is obtained from bending the covariance matrices because 
of the constraints imposed by special properties of the correlation matrices. However, changes in the more reliable elements, such as elements $(1,2)$, and $(2,3)$, are at most about $2 \%$.

It has been speculated that weighted bending, by utilization of some measure of reliability (reflecting degrees of freedom in a residual maximum likelihood estimation process) may bring disparately estimated correlations closer to the their joint residual maximum likelihood estimate. This might be true, however, answering this question is beyond the scope of the present study.

\section{Implementation Issues}

From a practical point of view several questions may be raised. One difficult question to address is which of the (co)variance or the correlation matrices should be used for bending. The answer very much depends on the amount and sources of information used in the formation of the original non-PD matrix. In some situations, e.g., international genetic evaluations of dairy cattle populations, variances are generally estimated from a very large number of observations, while estimation of covariances is based on few observations, not to mention country combinations whose correlations are estimated only based on pedigree information. In such cases, working with correlations may be preferred. In other cases, variances and covariances within blocks of the original non-PD matrix may be equally reliable and one may wish to keep them in accordance with each other by treating variances and covariances equally, i.e., by bending (co)variance matrices. If there are no external clues available to help, one may wish to use (co)variance matrices, because the proposed algorithm for them is simpler. Another question is whether the position of each trait in the correlation matrix has any effect on the outcome of the bending. In several examples that we have investigated carefully, position of the traits had no effect on their final values.

The choice of values for the scalar $a$ is crucial to both unweighted and weighted bending. If $a<1.0$ this leads to the same result as $a=1.0$; however, convergence is achieved at a slower rate. The effect of $a>1.0$ is rather complicated and depends also on the value of the weighting coefficient matrix $(\mathbf{C})$, but in general it can be said that $a>1.0$ eventually leads to faster convergence to the PD matrix but also to the divergence of values in the (co)variance or correlation matrix.

Despite the large number of iterations necessary to arrive at weighted bended matrices, the computational demand of implementing these algorithms is always negligible because the matrices involved are very small. We have tested the above algorithm in the international genetic evaluations of dairy bulls at the Interbull Centre for (co)variance/correlation matrices of up to $26 \mathrm{Hol}-$ stein populations. Number of iterations needed to achieve the weighted bending may be as large as several thousand. However, we never encountered failure to converge and convergence never required more than a few seconds. In our experience, preparing matrices of the weighting factors, $\mathbf{W}$, has been more demanding than the actual bending process.

\section{ACKNOWLEDGMENTS}

H. Jorjani would like to acknowledge financial support from USDA/NAAB.

\section{REFERENCES}

Árnason, T. 1984. Genetic studies on conformation and performance of Icelandic Toelter Horses. II. Construction of Multi-trait selection indices and modification of covariance matrices by the "bending" method. Acta Agric. Scand. 34:428-439.

Essl, A. 1991. Choice of an appropriate bending factor using prior knowledge of the parameters. J. Anim. Breed. Genet. 108:89-101.

Hayes, J. F., and W. G. Hill. 1981. Modification of estimates of parameters in the construction of genetic selection indices ('bending'). Biometrics 37:483-493.

Henshall, J. M., and K. Meyer. 2002. "PDMATRIX"-Programs to make matrices positive definite. Proceedings of the 7th World Congress on Genetics Applied to Livestock Production. Communication No. 28-12. Vol. 33:753-754.

Jorjani, H., L. Klei, and U. Emanuelson. 2002. Combining disparately estimates of genetic correlations. Interbull Bull. 29:1-3.

Klei, L., E. J. Pollak, and R. L. Quaas. 1988. Genetic and environmental parameters associated with linearized type appraisal scores. J. Dairy Sci. 71:2744-2752.

Meuwissen, T. H. E., and E. Kanis. 1988. Application of bending theory in a pig-breeding situation. Livest. Prod. Sci. 18:85-91.

Meyer, K., and Hill, W. G. 1983. A note on the effects of sampling errors on the accuracy of genetic selection indices. J. Anim. Breed. Genet. 100:27-32. 